\title{
Hands are vehicles for transmission of Streptococcus pneumoniae in novel controlled human infection study
}

\author{
To the Editor:
}

Streptococcus pneumoniae (pneumococcus) is a major cause of acute otitis media, sinusitis, pneumonia and meningitis worldwide [1]. More than 1.2 million infant deaths are attributed to S. pneumoniae annually [2].

Colonisation of the human nasopharynx with pneumococcus is common, with prevalence between $40 \%$ and $90 \%$ in children $<5$ years old and approximately $10 \%$ in adults in cross-sectional surveys [3]. Colonisation is important as it is a pre-requisite to infection, the primary reservoir for transmission, but can also boost immunity $[1,4]$.

It is postulated that transmission of $S$. pneumoniae occurs primarily by indirect contact via inhalation of airborne droplets. Supporting this are observations that transmission is associated with higher density living (day care centres, prisons and nursing homes), and is enhanced by concurrent viral respiratory tract infections [5]. For upper respiratory tract infections in general, direct contact is implicated in disease transmission, which can be interrupted by hand washing [6]. However, the relative contribution of direct and indirect transmission modes to pneumococcal colonisation and disease are unknown. We aimed to assess the potential for pneumococcal hand-to-nose transmission to cause nasopharyngeal colonisation.

We enrolled 63 healthy adult participants, between April and May 2017, to our controlled Experimental Human Pneumococcal Challenge model (EHPC), modified slightly from previous published protocols to assess "hand-to-nose" [7].

Briefly, participants were administered pneumococcus $\left(3.2 \times 10^{6}\right.$ mid-log phase colony-forming units $(\mathrm{CFU})$ of $S$. pneumoniae serotype 6B prepared as previously described) onto their fingertip or dorsum of their hand [7]. Bacterial density was confirmed by serial dilutions of the bacterial stock on blood agar (Oxoid, Basingstoke, UK). Participants were asked to either sniff the bacterial residue or to make direct contact with the nasal mucosal surface (pick/poke their nose), and to do so either immediately after exposure ("wet") or when visibly dry (approximately 1-2 min after exposure). Baseline screening was undertaken prior to pneumococcal exposure; participants who were naturally colonised with pneumococcus were excluded prior to exposure. Pre-exposure throat swabs were also assayed for respiratory viruses (adenovirus, influenza A and B, coronavirus, respiratory syncytial virus/human metapneumovirus, rhinovirus and parainfluenza 1-4), using multiplex PCR as previously published [8].

All participants were followed up for 9 days after exposure. Nasopharyngeal colonisation was assessed in nasal washes collected at days 2, 6 and 9. Pneumococcal density in nasal washes was determined by culture in real time; serotype was determined as $6 \mathrm{~B}$ using a latex agglutination kit (Statens Serum Institute, Copenhagen, Denmark). Following completion of the study all samples were tested by quantitative PCR (qPCR) with primers for lytA, and for S. pneumoniae serotype 6A/B. Rates of pneumococcal acquisition between groups were compared using Fisher's exact test.

All participants gave written, informed consent. Ethical permission was granted by the local National Health Service Research Ethics Committee (17/NW/0054).

@ERSpublications

Hands can be vehicles for of transmission of pneumococcus leading to nasopharyngeal colonisation, even after drying http://ow.ly/svlu30liKqP

Cite this article as: Connor V, German E, Pojar S, et al. Hands are vehicles for transmission of Streptococcus pneumoniae in novel controlled human infection study. Eur Respir J 2018; 52: 1800599 [https://doi.org/10.1183/13993003.00599-2018]. 
Between March and June 2017, 76 participants were recruited for the hand to nose study (ISRCTN: 12909224), with 63 participants completing all the study visits. The mean age of volunteers was 22.6 years of age (range 18-45, median 21 years) and the male:female ratio was 23:40.

40 participants were initally randomly allocated to four different transmission groups: 1) sniffing wet bacterial suspension (“wet sniff”); 2) sniffing bacterial suspension after air-drying (“dry sniff”); 3) pick/ poke nose with finger exposed to wet bacterial suspension ("wet poke"); and 4) pick/poke nose with finger exposed to bacterial suspension after air-drying ("dry poke").

In this initial cohort of 40 participants, eight (20\%) were found to be experimentally colonised with pneumococcus (6B) at follow-up visits by culture, with highest rates in the "wet poke" (4/10,40\%), and "wet sniff" $(3 / 10,30 \%)$ groups. Allowing the bacteria to dry on the skin before "sniff" or "poke" led to $1 / 10$ and $0 / 10$ participants becoming colonised respectively (figure 1). The four different groups were too small to compare, analysis of the wet versus dry groups highlighted that colonisation rates in the "wet" group were significantly higher than the "dry" group ( $\mathrm{p}=0.04$, Fisher exact test).

Median post-exposure colonisation densities (CFU per mL nasal wash) of the "wet sniff", "wet poke" and "dry sniff" groups were $5.6 \times 10^{1}$ (range $4.3 \times 10^{-1}$ to $3.7 \times 10^{6}$ ), 4.7 (range $4.5 \times 10^{-1}$ to $1.3 \times 10^{2}$ ) and 2.4 (range 1.2 to 9.6 ) respectively. Following the culture results the "wet sniff" group was expanded to improve precision estimates of rates, this group was expanded due to the combination of good colonisation rates and high density of colonisation episodes in participants. A total of $6 / 33$ volunteers became colonised (18\%). No viruses were detected in any throat swabs taken before pneumococcal exposure.

Molecular detection (lytA qPCR) identified higher colonisation rates compared with culture (whole cohort evaluated: $33 / 63$ (52\%) versus 13/63 (23\%), respectively; $\mathrm{p}<0.0001$, Fisher's exact test) (figure 1 ). This was most apparent in "dry poke" group; colonisation in $0 / 10$ by culture and $7 / 10$ using qPCR. Samples which were only positive with qPCR tended to have lower densities of carriage compared to samples positive with both methods. Higher densities were noted in the wet sniff group and in samples that were carriage positive with both methods.

The study shows for the first time, using a controlled human infection model, that the hands can be a vehicle for transmission of pneumococcus and lead to acquisition of nasopharyngeal colonisation.

This novel use of a human challenge model allowed for the study of pneumococcal transmission and colonisation in a controlled environment. Previous pneumococcal challenge studies have directly inoculated bacteria into the noses of participants. This direct exposure method with $6 \mathrm{~B}$ serotype has resulted in a colonisation rate of approximately $45 \%$ using culture detection method $[3,4]$. Colonisation rates in human challenge models are probably dependent on a variety of host and bacterial factors including host immunity. Lower colonisation rates in this study may be due to the sniffing transmission

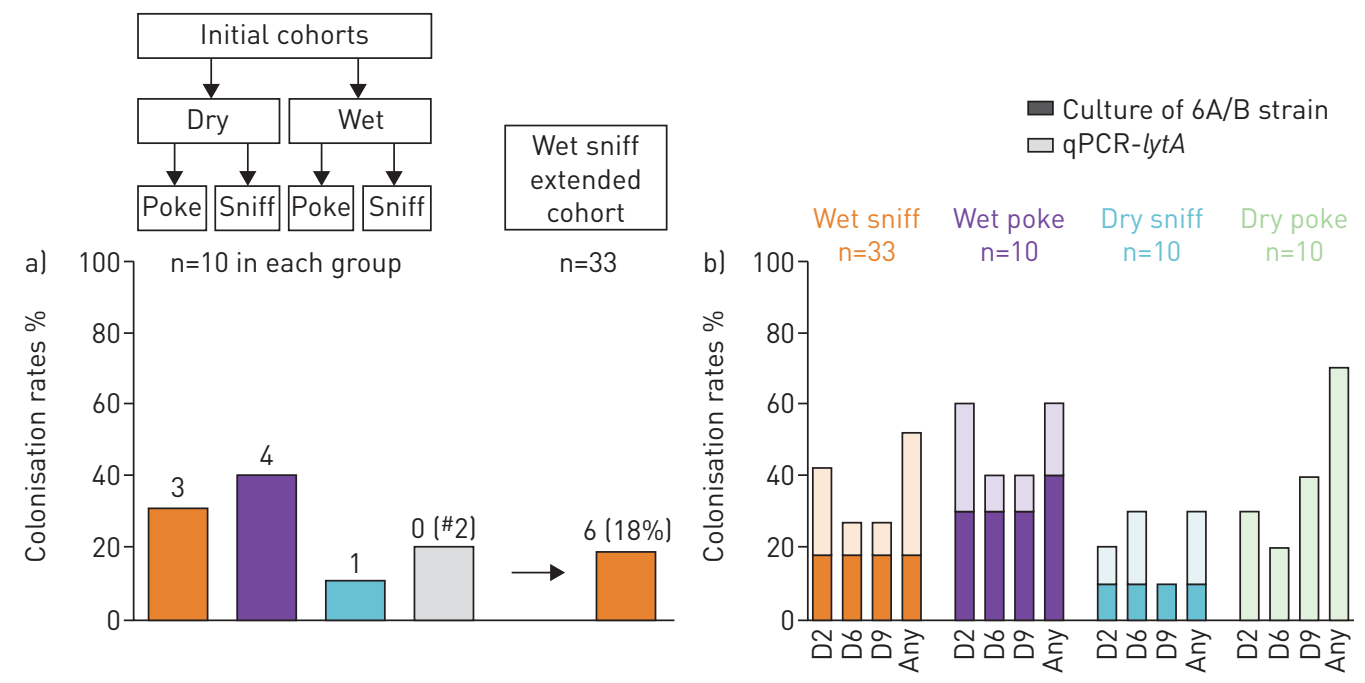

FIGURE 1 a) Colonisation rates following classical culture of nasal wash samples at any time point after exposure in each transmission group. " : no experimental carriers of strain 6B found in dry poke group but two participants became natural carriers of pneumococci after exposure to 6B. b) Comparison of culture (6A/B strain only) and lytA quantitative PCR (qPCR) results for different transmission methods. 
method causing movement of the pathogen out of the nasopharynx or due to death of pneumococcal bacteria during the drying process on the hands.

Pneumococcal survival on the skin has been reported, with persistence of viable bacteria for $3 \mathrm{~h}$ following exposure [9]. We have further shown that pneumococcus can survive drying on the skin, and go on to cause colonisation. An association between frequent sharing of drinking glasses or bottles and pneumococcal colonisation has also been reported [10]. The authors suggested that these findings indicate pneumococcal transmission through saliva is a highly plausible route in young adults, however, the possibility that the hands could be vectors for this transmission was not considered or investigated. Following the results of this study, it is plausible that hand contamination and hand-to-nose transmission could be implicated in the higher rates of colonisation seen in the "frequently sharing group".

Our results support other studies in suggesting that determining pneumococcal colonisation by both culture and qPCR are complementary [11, 12]. Higher rates of detection using molecular methods likely represent superior sensitivity at low-density colonisation. Of those only detectable by qPCR, all had densities below $10^{3}$ copies per $\mathrm{mL}$. The main limitation is the inability to distinguish viable from non-viable bacteria, potentially causing false-positive results [3].

Limitations of the study include the use of a young healthy adult population. Children are the primary reservoirs for community pneumococcal transmission, although an adult population was used in this study, the results suggest the potential for hand-to-nose transmission in paediatric populations [2]. The short follow-up period of participants limits our ability assess how duration of colonisation and different transmission methods affect longevity of colonisation. The bacteria stock used for exposure is derived from culture media and is mid-log phase; these experimental conditions may influence pneumococcal viability and colonisation potential. Finally, we only investigated the potential transmission of pneumococcus by self-inoculation; examining relative risk of pneumococcal spread via aerosolised bacteria, direct contact or indirect contact would be beneficial, similar to previous studies investigating transmission of respiratory viruses [13].

We were unable to investigate the relationship between colonisation acquisition and concurrent viral infection due to the absence of viral infection in our participants, probably due to seasonality. In a previous study, asymptomatic co-infection increased the odds of experimental pneumococcal colonisation ( $75 \%$ virus positive participants became colonised versus $46 \%$ virus negative; $\mathrm{p}=0.02$ ), and other experiments have shown that viruses (such as rhinovirus) facilitate acquisition and transmission between individuals $[8,14]$.

This study is the first to show that direct pneumococcal transmission can occur leading to colonisation acquisition. Good hand hygiene practices, already known to reduce enteric bacterial and viral disease, may also prevent the spread of bacteria thought to be primarily spread through aerosolisation. Follow-on experimental trials would be needed to examine whether true person-to-person transmission could occur with pneumococcus moving from the hands into the nose efficiently enough to cause colonisation. This supports advice about cross-infection risk in patients with bronchiectasis from Chalmers et al. [15]. Advising our high-risk patients to practise rigorous hand hygiene, to avoid shaking of hands and ensure basic infection control measures, such as avoidance of sharing food, drink and mobile phones, may reduce the transmission of respiratory bacterial pathogens such as pneumococcus. For healthy adults and children, it is unclear if reducing exposure to pneumococcus would be beneficial for overall health; pneumococcal exposure and colonisation have been shown to elicit immune responses which may be beneficial in later life $[4,16]$.

Better understanding of the duration of survival of pneumococci in nasal secretions on the hands and the frequency of hand contamination would help understanding of the transmission process further. Studies to improve knowledge about the process of pneumococcal shedding from the nose in humans during colonisation, and factors which promote shedding, are also warranted. This modification of the EHPC model has several potential uses, including testing of current or new hand cleaning interventions to ensure reduction in transmission of this important bacterial pathogen.

Victoria Connor ${ }^{1,2}$, Esther German ${ }^{1}$, Sherin Pojar ${ }^{1}$, Elena Mitsi $^{1}$, Caroline Hales ${ }^{1,2}$, Elissavet Nikolaou ${ }^{1}$, Angela Hyder-Wright ${ }^{1,2}$, Hugh Adler ${ }^{1}$, Seher Zaidi ${ }^{1}$, Helen Hill ${ }^{1}$, Simon P. Jochems ${ }^{1}$, Hassan Burhan ${ }^{2}$, Neil French ${ }^{3}$, Timothy Tobery ${ }^{4}$, Jamie Rylance ${ }^{1}$ and Daniela M. Ferreira ${ }^{1}$

${ }^{1}$ Liverpool School of Tropical Medicine, Liverpool, UK. ${ }^{2}$ Royal Liverpool University Hospital, Liverpool, UK. ${ }^{3}$ University of Liverpool, Liverpool, UK. ${ }^{4}$ Unilever, CT, USA.

Correspondence: Victoria Connor, Liverpool School of Tropical Medicine, Liverpool Life Sciences Accelerator, 1 Daulby Street, Liverpool L7 8XZ, UK. E-mail: victoria.connor@lstmed.ac.uk

Received: March 272018 | Accepted after revision: Aug 012018 
This study is registered at ISRCTN with identifier 12909224.

Conflict of interest: V. Connor reports grants from Unilever, during the conduct of the study; the study was part-funded by Unilever, who participated in the study design. The conduct of the study, analysis and presentation of results, and decision to publish were solely determined by the authors, without influence from any funding source. E. German reports grants from Unilever and Medical Research Council, during the conduct of the study. S. Pojar reports grants from Unilever and MRC, during the conduct of the study. E. Mitsi has nothing to disclose. C. Hales reports grants from Unilever, during the conduct of the study; the study was part-funded by Unilever, who participated in the study design. The conduct of the study, analysis and presentation of results, and decision to publish were solely determined by the authors, without influence from any funding source. E. Nikolaou has nothing to disclose. A. Hyder-Wright reports grants from Unilever, during the conduct of the study; the study was part-funded by Unilever, who participated in the study design. The conduct of the study, analysis and presentation of results, and decision to publish were solely determined by the authors, without influence from any funding source. H. Adler reports grants paid to institution from Unilever, during the conduct of the study. S. Zaidi has nothing to disclose. H. Hill has nothing to disclose. S.P. Jochems has nothing to disclose. H. Burhan has nothing to disclose. N. French reports grants (paid to the University of Liverpool) from Unilever PLC, during the conduct of the study. T. Tobery is a full time employee of Unilever, which provided funding for this work; however, T. Tobery's compensation from Unilever is not affected by the work described here or its publication. J. Rylance has nothing to disclose. D.M. Ferreira has nothing to disclose.

Support statement: This work received financial support from Unilever and The Medical Research Council (MRC). Funders did not have any input into the study design. Funding information for this article has been deposited with the Crossref Funder Registry.

Acknowledgements: The authors thank the respiratory research team; trial steering committee members; Stephen Gordon, Malawi-Liverpool-Wellcome Trust Clinical Research Programme; clinicians who provided safety on-call cover; John Blakey, Steve Aston and Ben Morton, and the Liverpool School of Tropical Medicine (LSTM) respiratory group.

\section{References}

1 Simell B, Auranen K, Kayhty H, et al. The fundamental link between pneumococcal carriage and disease. Expert Rev Vaccines 2012; 11: 841-855.

2 van der Poll T, Opal SM. Pathogenesis, treatment, and prevention of pneumococcal pneumonia. Lancet 2009; 374: 1543-1556.

3 Gritzfeld JF, Cremers AJ, Ferwerda G, et al. Density and duration of experimental human pneumococcal carriage. Clin Microbiol Infect 2014; 20: O1145-O1151.

4 Ferreira DM, Neill DR, Bangert M, et al. Controlled human infection and rechallenge with Streptococcus pneumoniae reveals the protective efficacy of carriage in healthy adults. Am J Respir Crit Care Med 2013; 187: 855-864.

5 Musher DM. How contagious are common respiratory tract infections? N Engl J Med 2003; 348: 1256-1266.

6 Roberts L, Smith W, Jorm L, et al. Effect of infection control measures on the frequency of upper respiratory infection in child care: a randomized, controlled trial. Pediatrics 2000; 105: 738-742.

7 Gritzfeld JF, Wright AD, Collins AM, et al. Experimental human pneumococcal carriage. J Vis Exp 2013; 72: 50115.

8 Glennie S, Gritzfeld JF, Pennington SH, et al. Modulation of nasopharyngeal innate defenses by viral coinfection predisposes individuals to experimental pneumococcal carriage. Mucosal Immunol 2016; 9: 56-67.

9 Smith-Vaughan H, Crichton F, Beissbarth J, et al. Survival of pneumococcus on hands and fomites. BMC Res Notes 2008; 1: 112 .

10 Levine H, Zarka S, Dagan R, et al. Transmission of Streptococcus pneumoniae in adults may occur through saliva. Epidemiol Infect 2012; 140: 561-565.

11 Chien Y-W, Vidal JE, Grijalva CG, et al. Density interactions between Streptococcus pneumoniae, Haemophilus influenzae and Staphylococcus aureus in the nasopharynx of young Peruvian children. Pediatr Infect Dis J 2013; 32: 72 .

12 Olwagen CP, Adrian PV, Madhi SA. Comparison of traditional culture and molecular qPCR for detection of simultaneous carriage of multiple pneumococcal serotypes in African children. Sci Rep 2017; 7: 4628.

13 Dick EC, Jennings LC, Mink KA, et al. Aerosol transmission of rhinovirus colds. J Infect Dis 1987; 156: 442-448.

14 Karppinen S, Terasjarvi J, Auranen K, et al. Acquisition and transmission of Streptococcus pneumoniae are facilitated during rhinovirus infection in families with children. Am J Respir Crit Care Med 2017; 196: 1172-1180.

15 Chalmers JD, Ringshausen FC, Harris B, et al. Cross-infection risk in patients with bronchiectasis: a position statement from the European Bronchiectasis Network (EMBARC), EMBARC/ELF patient advisory group and European Reference Network (ERN-Lung) Bronchiectasis Network. Eur Respir J 2018; 51: 1701937.

16 Wright AK, Bangert M, Gritzfeld JF, et al. Experimental human pneumococcal carriage augments IL-17A-dependent T-cell defence of the lung. PLoS Pathog 2013; 9: e1003274. 